\title{
Perceptions of Recovery and Satisfaction in the Short Term After Orthognathic Surgery
}

\author{
Ceib Phillips, MPH, PhD* [Research Professor], H. Asuman Kiyak, MA, $\mathrm{PhD}^{\dagger}$ [Professor], \\ Dale Bloomquist, DDS, MS ${ }^{\ddagger}$, and Timothy A. Turvey, DDS, MS $\$$ [Professor] \\ "Department of Orthodontics, School of Dentistry, University of North Carolina, Chapel Hill, NC \\ tDepartment of Oral and Maxillofacial Surgery, University of Washington, Seattle, WA \\ ‡Private Practice, Seattle, WA \\ §Department of Oral and Maxillofacial Surgery, University of North Carolina, Chapel Hill, NC
}

\begin{abstract}
Purpose-A 2-arm, parallel-group, stratified-block, randomized clinical trial was designed to assess whether patients' perceptions of recovery and satisfaction 4 to 6 weeks after surgery were affected by 3 factors: preparation strategy (viewing a visual treatment simulation), attitudes (expectations about recovery), and psychologic distress (reported before surgery).

Patients and Methods-One hundred eighty-four patients with a dentofacial disharmony scheduled for orthognathic surgery were randomly assigned to 1 of 2 preparation strategy groups: a standard presurgical consultation with or without a computerized treatment simulation presentation. Psychologic well-being and expectations regarding recovery were obtained before surgery and perceptions of recovery, and satisfaction were assessed for 126 patients at 4 to 6 weeks after surgery.
\end{abstract}

Results-Viewing a treatment simulation before surgery did not affect patients' perceptions of post-surgical discomfort or satisfaction at 4 to 6 weeks after surgery. Patients who overestimated the discomfort or problems they would experience reported significantly lower average level of problems than those who did not overestimate. Patients who were psychologically distressed before surgery reported, on average, significantly more discomfort or difficulty with symptoms, social/self-concerns, general health, and overall recovery after surgery.

Conclusion-Viewing a treatment simulation before surgery does not, on average, negatively affect perception of symptoms or satisfaction 4 to 6 weeks after surgery. Orthognathic surgery patients who are psychologically distressed before surgery tend to report a higher recovery burden overall and, on average, experience more difficulty with symptoms, social/self-concerns, and general health in the first 1 or 2 months after surgery.

Patients' perceptions of and satisfaction with the outcomes of orthognathic surgery are influenced by their presurgical expectations and psychologic well-being. Excessively negative expectations regarding the recovery period may heighten a patient's anxiety and stress, which can slow recovery, but predictable events cause less emotional response than unpredictable ones. ${ }^{1}$ Dissatisfaction, negative mood, and/or anxiety postsurgery are more likely to be expressed by surgery patients if some "unexpected" event happened. For example, experiencing more pain or swelling than had been anticipated, ${ }^{2-4}$ or the feeling of 
being "unprepared" for treatment may result in negative outcomes. ${ }^{5-7}$ Recent findings indicate that presurgical psychologic distress has a negative impact on postsurgical patient outcomes as well as on their perceived oral health before and after surgery. ${ }^{8}$

Reports from 2 randomized clinical trials provide evidence that patient preparation for orthognathic surgery can affect both short- and long-term expectations. ${ }^{9,10}$ Patients who viewed a visual treatment simulation before treatment, a simulated computer image of their profile after surgery, tended to have elevated expectations of the improvement that would occur in their self-image as a result of treatment. ${ }^{9}$ The effect on expectations for those who viewed a simulation after starting orthodontic treatment, only 1 or 2 months before surgery, was not as straightforward. Viewing a treatment simulation shortly before surgery did not affect, on average, expectations about treatment outcome but did influence expectations about short-term recovery problems and concerns. ${ }^{10}$ This effect on short-term expectations was moderated by the patient's psychologic distress. Viewing a treatment simulation tended to increase concern about the problems that might be experienced after surgery for those individuals who were not psychologically distressed. Patients who did report experiencing psychologic distress before surgery seemed to benefit from viewing a treatment simulation. These patients reported less concern about possible postsurgical problems compared to those who were distressed and did not view a treatment simulation. ${ }^{10}$

These observations led to the inclusion of expectations and presurgical psychologic distress as explanatory variables in the analysis of the postsurgical patient perceptions of recovery from the 2-arm, parallel-group, stratified-block, randomized clinical trial designed to evaluate the effect of viewing a visual treatment simulation before surgery. The intent of this project was to assess whether patients' perceptions of recovery and satisfaction 4 to 6 weeks after surgery were affected by 3 factors: preparation strategy (viewing a visual treatment simulation), attitudes (expectations about recovery), and psychologic distress (reported before surgery). Our primary hypothesis was that viewing a treatment simulation presentation shortly before surgery would not have a sustained effect after surgery.

\section{Patients and Methods}

\section{Subjects}

Participation was limited to patients who were scheduled for a presurgery consultation appointment between July 1995 and June 1999 in the Department of Oral and Maxillofacial Surgery at the University of North Carolina (UNC) or in a community-based private practice in Seattle, WA. Patients between 15 and 50 years of age with a developmental anteroposterior and/or vertical facial skeletal problem were eligible. Patients with dentofacial asymmetry, congenital defects, or traumatic injury were excluded.

Patients were invited to participate at their initial presurgery consultation appointment. All study procedures were approved by the Institutional Review Board at the UNC and the University of Washington, and all participating subjects (and their legal guardians if <18 years old) gave written informed consent for their participation. A total of 220 patients (83 from Seattle and 137 from UNC) consented to participate. One hundred eighty-four were randomly allocated to a preparation strategy using a stratified block randomization with center (UNC and Seattle) and gender (male and female) as strata with a block size of 8 . Within each stratum, patients were allocated to a standard case presentation or a standard plus individualized treatment simulation presentation group according to a randomization sequence prepared using Proc Plan. ${ }^{11}$ Assignment to preparation strategy was obtained from the study website after a patient was enrolled. To ensure a common knowledge base, patients viewed a videodisc (Videodiscovery Inc, Seattle, WA, 1987) before the case presentation. This program prepares patients by providing information about their upcoming 
surgery and postsurgery recovery: diet, anesthesia, pain and swelling, and other potential problems.

For patients allocated to the treatment simulation presentation group, one individualized surgical simulation was constructed from a digital image of the patient's profile obtained at the initial presurgery consultation appointment by the attending orthodontist and/or surgeon and shown to the patient by a research associate 1 to 2 months before surgery. A checklist was used to ensure the information communicated to the 2 groups of patients about the effect of surgery was as similar as possible. Patients were asked to complete a set of selfreport questionnaires about their perceptions of recovery, satisfaction, and psychologic wellbeing shortly before and 4 to 6 weeks after surgery.

\section{Patient Self-Report Variables}

Psychologic Distress (SCL-90R)—Patients were asked to complete the Symptom Checklist-90 Revised (SCL-90R) $)^{12}$ before the presurgery consultation appointment and 4 to 6 weeks after surgery (Table 1). The SCL-90R is a 90-item self-report questionnaire used to assess the current level of psychologic distress. The patient responds to the question, "Within the past 7 days, how much were you distressed by ...?" for each item using a 5point scale from "not at all (0)" to "extremely (4)." An overall psychologic distress measure, global severity index (GSI), was calculated and transformed, using the non-psychiatric gender- and age- (adolescent versus adult) specific patient norms, to a standardized T-score (T-GSI). The T-GSI combines information on both the number of symptoms and the intensity of perceived distress. Another indication of the level of current psychologic distress is represented by "positive diagnosis," an epidemiologic screening measure for psychiatric disorder. An individual was considered as having a clinically significant level of distress if the T-GSI score was above 63 or if 2 dimensions on the SCL-90R exceeded a T-score of 63. "Positive diagnosis" represents an elevation in distress that serves as an indicator for referral to a mental health professional. ${ }^{12}$

Presurgical Expectations (Short-Term Expectations)-Two to 4 weeks after the presurgery consultation but before surgery, each patient completed the Short-Term Expectations (STE). ${ }^{10}$ This questionnaire, adapted from Kiyak et al's ${ }^{3}$ conceptualizations, assesses the discomfort or difficulty expected during the first month after surgery. Each item was rated using a 7-point scale from "expect no discomfort (1)" to "expect much discomfort (7)." The 3 subscales for STE were labeled postsurgical discomfort, social well-being concerns, and general health. An average total score and average subscale scores were calculated. The higher the score, the higher was the expectation of discomfort or problem.

Postsurgical Perceptions-Four to 6 weeks after surgery, patients completed a questionnaire, adapted from Kiyak et al, ${ }^{3}$ to assess Postsurgical Perceptions (PSP) about the recovery process and the level of current discomfort/difficulty. The PSP contains all of the items on the STE as well as an additional 5 items in the dentofacial appearance subscale. Four subscales of recovery, identified by a factor analysis, are symptom recovery, dentofacial appearance, general health recovery, and social/self recovery. These subscales are derived from 26 items that measure the postsurgical level of discomfort or difficulty experienced in the past month on a 7-point scale ranging from "none (1)" to "great deal (7)." An average total score and average subscale scores, based on the items comprising each subscale, were calculated. The higher the score, the more problems or difficulties are experienced by the patient. In this sample, Cronbach's $a$ ranged from 0.74 to 0.92 for PSP.

Predictability-The discordance between the anticipated extent of discomfort or problem and the level experienced was calculated as the difference between the average PSP score 
and the average STE score for the total and each of the subscales. Because there was no corresponding dentofacial appearance subscale on the STE, the anticipatory level for social concerns on the STE was used to calculate the discrepancy for both the social/self recovery and dentofacial appearance PSP sub-scales. The differences in the before surgery expectation and the after surgery perception scores were dichotomized into 2 "predictability" categories for the total and each subscale. The 2 predictability categories were overestimation, overestimating (STE > PSP) by at least 1 unit; and nonoverestimation, concordance (STE $=$ PTP) or underestimating (STE < PSP) by at least 1 unit the level of discomfort that would be experienced.

Satisfaction-Satisfaction With Treatment (SAT) assesses satisfaction with surgery, recovery, and outcome. Items were constructed as positive and negative statements to avoid response bias. Negative items were reverse keyed after data entry. Three dimensions of satisfaction were identified from the 25 items: preparation/knowledge, self and interpersonal outcomes, and functional outcomes. Each item was presented as a statement rated on a 7point scale from "disagree strongly (1)" to "agree strongly (7)." An average total score and average subscale scores were calculated. The higher the score, the more positive was the satisfaction level. In this sample, Cronbach's $a$ ranged from 0.82 to 0.88 for SAT.

Patients were paid $\$ 10$ if they returned all questionnaires at the 4- to 6-week postsurgery assessment. Patients were encouraged but not required to answer all items on each questionnaire. If more than 2 items for a given subscale were skipped, the subscale score was set to missing.

\section{Data Analysis}

The equivalence of the participants in the 2 preparation groups and the 2 centers was assessed with Mantel-Haenszel $\chi^{2}$ statistics for nominal characteristics and an unpaired $t$ test for age at surgery. The effects of the treatment simulation, positive diagnosis for psychologic distress at the presurgery visit, and overall "predictability" on the total score of the PSP and SAT and each of the PSP and SAT dimension scores were analyzed using factorial analysis of variance (ANOVA). Center and gender were maintained as main effects in all models because these were stratification factors in the randomization. All possible pairwise interaction terms between the explanatory variables were included in the initial model. Interactions with values of $P>.10$ were eliminated and reduced models were analyzed. Level of significance was set at .01 for all bivariate analyses and final ANOVA models.

Pearson correlation coefficients were calculated to examine the simple bivariate relationships among the following variables: age at the time of surgery, psychologic distress (T-GSI) before surgery and at 4 to 6 weeks, expectations regarding the short-term recovery period, perception of problems/difficulties during the short-term recovery period, and satisfaction.

\section{Results}

\section{Subject Attrition}

Of the initial sample of 220, 184 patients completed the presurgery data collection protocol. Fifty-five (68\%) subjects from Seattle and $71(70 \%)$ from UNC completed the 4- to 6-week postsurgery data collection phase (Fig 1). The primary reason for missing data at this visit was the difficulty in coordinating clinical and research appointment times. Patients who were not seen at 4 to 6 weeks were similar to those who completed the baseline data collection phase in age $(P=.73)$, gender $(P=.36)$, race $(P=.18)$, percent randomized to a 
treatment simulation $(P=.15)$, and percent with elevated presurgical psychologic distress ( $P$ $=.46)$.

\section{Participants}

Participants were predominantly white (88\%) and female (72\%). Patients at the 2 centers were very similar in their demographic characteristics (Table 2), except that the percentage of patients older than 25 was higher in Seattle $(P=.002)$. The patients in Seattle ranged in age from 15 to 52 years and the median age was 32 years, whereas at UNC, the age range was 15 to 53 years but the median age was only 21 years. The characteristics of the patients randomized to the 2 presentation strategies were similar except that a higher percentage of the standard presentation group were not white (18\% versus $5 \%)$ and a higher proportion reported psychologic distress (30\% versus 14\%) (Table 2).

\section{Psychologic Distress}

After surgery, approximately the same percentage of patients (20\%) were psychologically distressed than before surgery (23\%). Interpersonal sensitivity issues were of concern both before (61\% of those distressed) and after (67\% of those distressed) surgery. Thirteen percent of the patients were distressed both before surgery and postsurgery.

\section{Perceptions of Postsurgical Problems}

At the 4- to 6-week postsurgical assessment, substantially more patients reported at least moderate discomfort related to symptom recovery than the proportion that reported problems with dentofacial appearance, general health, or social interactions (Table 3). Even with rigid fixation, more than half of the patients were still experiencing discomfort with biting and chewing. Half were at least moderately concerned with residual numbness, and $35 \%$ were concerned with residual facial swelling. Very few of the patients $(<15 \%)$ reported more than mild problems with being out in public or with social or interpersonal behaviors. Age at the time of surgery was not statistically correlated to the overall perception of problems or concerns (Table 4).

\section{Predictability}

Overall, the patients were well prepared for the level of problems or difficulties they would experience during the short-term recovery period. Sixty-nine percent reported the average total level of discomfort/concern experienced after surgery to be at least 1 unit less than what they had expected. For $30 \%$ of the patients, the average postsurgery recovery score and the average expectation score were within 1 unit. Less than $1 \%$ of the patients underestimated by at least 1 unit the extent of the problem or difficulty they would experience in specific domains: appearance, recovery symptoms, social interactions, or general health.

\section{Satisfaction}

The majority of patients were neutral, neither positive nor negative, about the preparation they had received for surgery or the outcomes of surgery (Table 5). Almost half of the patients agreed they had been emotionally prepared for surgery and were not surprised at how long it took to recover. Interestingly, $24 \%$ of the patients expected more improvement in their appearance and even more (37\%) were negative about the reactions of others to the change. In general, younger patients tended to report higher levels of satisfaction with the self/interpersonal and functional outcomes of surgery. However, the variability in satisfaction explained by age was quite minimal. The level of concern about recovery that the patient reported before surgery was not associated with the patient's satisfaction at 4 to 6 weeks after surgery (Table 4). 
Patients who expressed higher levels of problem or discomfort with their dentofacial appearance and/or social relations tended to report less satisfaction with the self/ interpersonal outcomes (Table 6). In addition, patients with higher symptom burdens reported lower satisfaction with the preparation they received and with their functional outcomes. Interestingly, patients with greater general health concerns and social interaction discomfort were less satisfied with the preparation they had received.

\section{Effect of Preparation Strategy and Center on Perceived Problems and Satisfaction}

Preparation strategy did not have a statistically significant effect on the average level of perceived problems in symptoms, dentofacial appearance, general health, or social/selfrecovery after surgery (Tables 7), nor were the average satisfaction scores significantly different for those who did and did not view a treatment simulation (Table 8). Differences in postsurgical perceptions of discomfort between the 2 centers were not statistically significant. However, satisfaction with interpersonal outcomes and overall satisfaction were significantly different between the 2 centers. On average, the university-based patients reported slightly higher satisfaction (interpersonal: mean $=4.13, \mathrm{SD}=1.22$ versus mean $=$ $5.04, \mathrm{SD}=1.20$ for community versus university setting, respectively; and overall mean = $4.68, \mathrm{SD}=0.82$ versus mean $=5.17, \mathrm{SD}=0.86$, respectively).

\section{Effect of Predictability on Perceived Problems and Satisfaction}

Predictability was dichotomized as patients who overestimated what they would experience by at least 1 unit versus those who did not overestimate. Interestingly, patients who overestimated the problems they would experience reported a significantly lower average level of problems overall and in all recovery domains-symptoms, social concerns, general health, and appearance — than those who did not overestimate (Table 7, Fig 2).

Although the average perception of postsurgical recovery burden was not statistically related to viewing a treatment simulation, the treatment preparation strategy may have an indirect effect because of the impact of viewing a simulation on the short-term expectations. The percentage of patients who overestimated the extent of the postsurgical problems they would experience was higher in the treatment simulation group compared with the standard preparation group (Fig 3).

The average satisfaction scores for preparation, function, and total satisfaction were not statistically different between those who overestimated the level of discomfort and those who did not (Table 8). However, the average interpersonal satisfaction score was significantly different. Those who overestimated the problems with social interactions after surgery expressed a higher average satisfaction (mean $=4.8 ; \mathrm{SD}=1.2$ ) with the selfimprovement and interpersonal outcomes than those who did not overestimate ( $\mathrm{SD}=1.3)$.

\section{Effect of Psychologic Distress on Perceived Problems and Satisfaction}

Higher levels of psychologic distress presurgery and postsurgery were associated with a higher level of perceived problems or discomfort overall and in social interactions (Table 4). Elevated psychologic distress at the 4- to 6-week follow-up explained approximately $10 \%$ of the variability in the level of perceived discomfort in all areas except dentofacial appearance, where it explained less than $5 \%$ of variability.

Patients who met the criteria for a "positive diagnosis" on the SCL-90R before surgery reported, on average, significantly more discomfort or difficulty with symptoms, social/selfconcerns, general health, and overall recovery (Table 7, Fig 4). 


\section{Discussion}

The age and demographic characteristics of the patients who participated in this study and were randomly assigned to the 2 surgical preparation strategies were representative of patients who have orthognathic surgery. ${ }^{13}$ Seventy percent of the enrolled subjects completed all data collection. There appears to be no bias in follow-up because the nonrespondents were similar in demographic and psychologic characteristics to those who completed the 4- to 6-week assessment.

Perception of recovery is affected by knowledge of the events and process of recovery ${ }^{2-4}$ and by how well the patient anticipates the emotional consequences of recovery events. ${ }^{5-7}$ For example, a patient may expect numbness after surgery but still not anticipate the discomfort or concern evoked by the sensation of not being able to tell if the cup is against his or her lips when drinking, or hypersensitivity while shaving. Only $31 \%$ of the patients in this study did not overestimate the postsurgical extent of problems they would experience. However, these patients reported a higher, on average, recovery burden than those who overestimated. The main anticipatory determinant appears to be whether the expectation exceeds the actual experience. As Johnston has said, "Wildly inaccurate but overestimated expectation ... [is better] than a mildly inaccurate but underestimated expectation." Expectations of problems with postsurgical recovery, social interactions, or general health issues were not associated with postsurgical concerns with appearance. This supports findings in medically compromised patients that expectations about treatment recovery affect the perception of signs and symptoms, not the perception of the outcome. ${ }^{14}$

Recent reports indicate that before orthognathic surgery, $18 \%$ to $33 \%$ of patients endorse clinically elevated levels of psychologic distress. ${ }^{10,15,16}$ In this sample, which includes academic and community-based practices and widely separated geographic regions, $13 \%$ of the patients were distressed both before and within the first 2 months after surgery, $9 \%$ were distressed only before surgery, and 7\% were distressed only after surgery. Presurgical levels of psychologic distress were not associated with overall satisfaction. This supports the findings of Hatch et al, ${ }^{16}$ who used a global satisfaction score based solely on willingness to undergo surgery again, to recommend surgery to a friend, and general satisfaction although such a global score is too general to assess specific components of satisfaction. Psychologic distress after surgery was associated with the satisfaction with the preparation for surgery and the average satisfaction with preparation score was marginally lower for those who reported distress before surgery.

Patients who were psychologically distressed before surgery reported significantly higher discomfort and higher symptom burdens at 4 to 6 weeks after surgery, as well as greater social and general health concerns. This finding supports previous reports that presurgical psychologic distress in orthognathic surgery patients has a negative impact on postsurgical outcomes $^{8}$ and on the perception of oral health before and after surgery. ${ }^{8}$ Substantial clinical research evidence indicates that patients who are psychologically distressed before surgery tend to have a slower postsurgical recovery, report more pain, and use more pain medication. ${ }^{14,17}$ These findings highlight the importance of improving patient management strategies for orthognathic surgery patients who display symptoms of psychologic distress presurgery. For a number of years, the debate regarding routine psychologic screening revolved around whether the orthognathic surgery patient population as a whole is "normal," rather than on the more important consideration of the psychologic well-being of the individual patient. Can optimal care can be given when psychologic well-being is not assessed? The present and previous studies indicate that a routine, standardized mental health screening process for patients before elective orthognathic surgery should be a routine 
part of the pretreatment/presurgery evaluation of a patient who is a candidate for orthognathic surgery.

Generally, patients are willing to complete an instrument such as the SCL-90R when they understand that 1) the clinician is interested in the patient's overall well-being, and not just the appearance of the teeth and/or face, and 2) the information is confidential and is meant to assist the clinician in supporting the patient during the treatment process. Psychologic distress, as indicated on a measure such as the SCL-90R, can alert the surgeon to explore those areas that may complicate or interfere with a patient's treatment and recovery from surgery. If appropriate, patients with clinically elevated scores can be referred to a mental health specialist for evaluation and counseling. A referral for counseling will generally be positively accepted by the patient if the clinician presents the referral as a constructive option for coping with the additional stress caused by surgery and recovery.

The primary objective of this study was to compare the short-term postsurgical effects of viewing a treatment simulation presented 1 to 2 months before surgery. Our results indicated no statistically significant differences in patients' perceptions of postsurgical symptom burden and satisfaction between those who viewed the treatment simulation and those who did not. This is not to say that viewing a treatment simulation does not affect patient perceptions of short-term recovery. The effect, if it exists, appears to be indirect. Viewing a treatment simulation shortly before surgery tends to increase concerns about the problems that might be experienced after surgery for those individuals who are not psychologically distressed before surgery, but decreases concerns in those who are distressed. ${ }^{10}$

Viewing a treatment simulation before surgery is likely beneficial regardless of the psychologic status of the patient. For those who are psychologically distressed before surgery, the concern about recovery may be excessive causing unnecessary anxiety and stress and viewing a treatment simulation tends to decrease the concern but not to such an extent that the expectation does not exceed the actual experience. For those who are not distressed, viewing a treatment simulation increases, on average, the level of concern to what may be a more realistic level. Viewing a treatment simulation after receiving information about the events and problems that might occur during the recovery process appears to be an effective cognitive strategy for providing patients, whether psychologically distressed or not, with a concrete, personalized impression of how recovery events might affect them.

The following conclusions can be drawn from this investigation:

1. Presentation of a treatment simulation 1 to 2 months before surgery does not directly affect patients' perceptions of discomfort/problems or their satisfaction in the short term after surgery.

2. Patients who reported elevated psychologic distress before surgery reported a significantly higher average level of problem or discomfort overall, as well as higher average dentofacial and social concern, and symptom and general health discomfort levels 4 to 6 weeks after surgery.

3. Patients who overestimated the problems or discomfort they would experience reported significantly lower average overall as well as lower average symptom recovery, social concern, and general health discomfort levels and lower average dentofacial appearance concern after surgery.

4. Neither predictability nor psychologic distress had an effect on overall satisfaction. Patients who were distressed before surgery tended to report less satisfaction with the preparation for surgery, whereas those who overestimated the perceived level of 
problems tended to report higher satisfaction with the interpersonal outcomes at 4 to 6 weeks after surgery.

\section{Acknowledgments}

The authors wish to thank Debora Price, Teresa Hagan, Will Harvey, Steve Dickens, Lawrence Patrick, and Carol

Dean for their assistance with data collection and processing.

This project was supported by National Institutes of Health grants DE10028 and DE13967.

\section{References}

1. Johnston M. Preoperative emotional states and postoperative recovery. Adv Psychosom Med. 1986; 15:1. [PubMed: 3706030]

2. Kiyak HA, McNeill RW, West RA, et al. Predicting psychologic responses to orthognathic surgery. J Oral Maxillofac Surg. 1982; 40:150. [PubMed: 6950057]

3. Kiyak HA, Vitaliano PP, Crinean J. Patients' expectations as predictors of orthognathic surgery outcomes. Health Psychol. 1988; 7:251. [PubMed: 3383832]

4. Ostler S, Kiyak HA. Treatment expectations versus outcomes among orthognathic surgery patients. Int J Adult Orthodon Orthognath Surg. 1991; 6:247. [PubMed: 1820409]

5. Garvill J, Garvill H, Kahnberg KE, et al. Psychological factors in orthognathic surgery. J Craniomaxillofac Surg. 1992; 20:28. [PubMed: 1564118]

6. Phillips C. Patient-centered outcomes: Impact on clinical practice and research. Semin Orthod. 1999; 5:223. [PubMed: 10860059]

7. Scott AA, Hatch JP, Rivera SM, et al. Psychosocial predictors of high-risk patients undergoing orthognathic surgery. Int J Adult Orthodon Orthognath Surg. 2000; 15:7. [PubMed: 11307227]

8. Hatch JP, Rugh JD, Clark GM, et al. Health-related quality of life following orthognathic surgery. Int J Adult Orthodon Orthognath Surg. 1998; 13:67. [PubMed: 9558537]

9. Phillips C, Hill BJ, Cannac C. The influence of video imaging on patients' perceptions and expectations. Angle Orthod. 1995; 65:263. [PubMed: 7486240]

10. Phillips C, Bailey L, Kiyak HA, et al. Effects of a computerized treatment simulation on patient expectations for orthognathic surgery. Int J Adult Orthodon Orthognath Surg. 2001; 16:87. [PubMed: 11482295]

11. SAS Institute, Inc. SAS/STAT User's Guide Version 7-1. Cary, NC: SAS Institute Inc; 1999. p. 2435-2462.

12. Derogatis, LR. SCL-90-R Symptom Checklist-90-R. Minneapolis, MN: National Computer Systems, Inc; 1994.

13. Bailey LJ, Haltiwanger LH, Blakey GF, et al. Who seeks surgical-orthodontic treatment: A current review. Int J Adult Orthodon Orthognath Surg. 2001; 16:280. [PubMed: 12390006]

14. Flood AB, Lorence DP, Ding J, et al. The role of expectations in patients' report of postoperative outcomes and improvement following therapy. Med Care. 1993; 31:1043. [PubMed: 7694013]

15. Phillips C, Bennett ME, Broder HL. Dentofacial disharmony: Psychological status of patients seeking treatment consultation. Angle Orthod. 1998; 68:547. [PubMed: 9851353]

16. Hatch JP, Rugh JD, Bays RA, et al. Psychological function in orthognathic surgical patients before and after bilateral sagittal split osteotomy with rigid and wire fixation. Am J Orthod Dentofacial Orthop. 1999; 115:536. [PubMed: 10229886]

17. Kravitz RL. Patient's expectations for medical care: An expanded formulation based on review of the literature. Med Care Res Rev. 1996; 53:3. [PubMed: 10156434] 


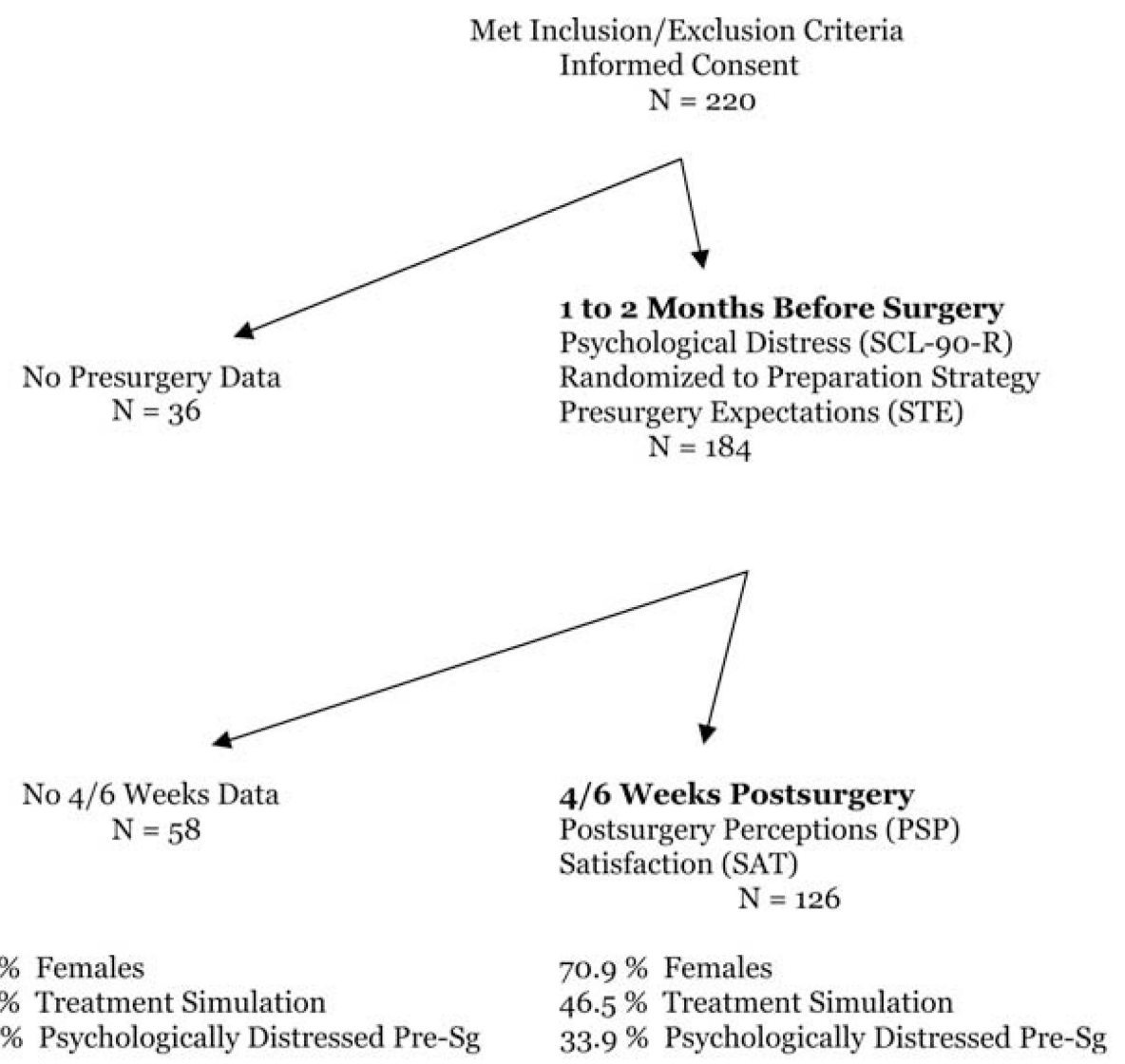

Figure 1.

Patient flow through the clinical trial and the demographic characteristics of those who completed the 4/6-week assessment and those who did not. 


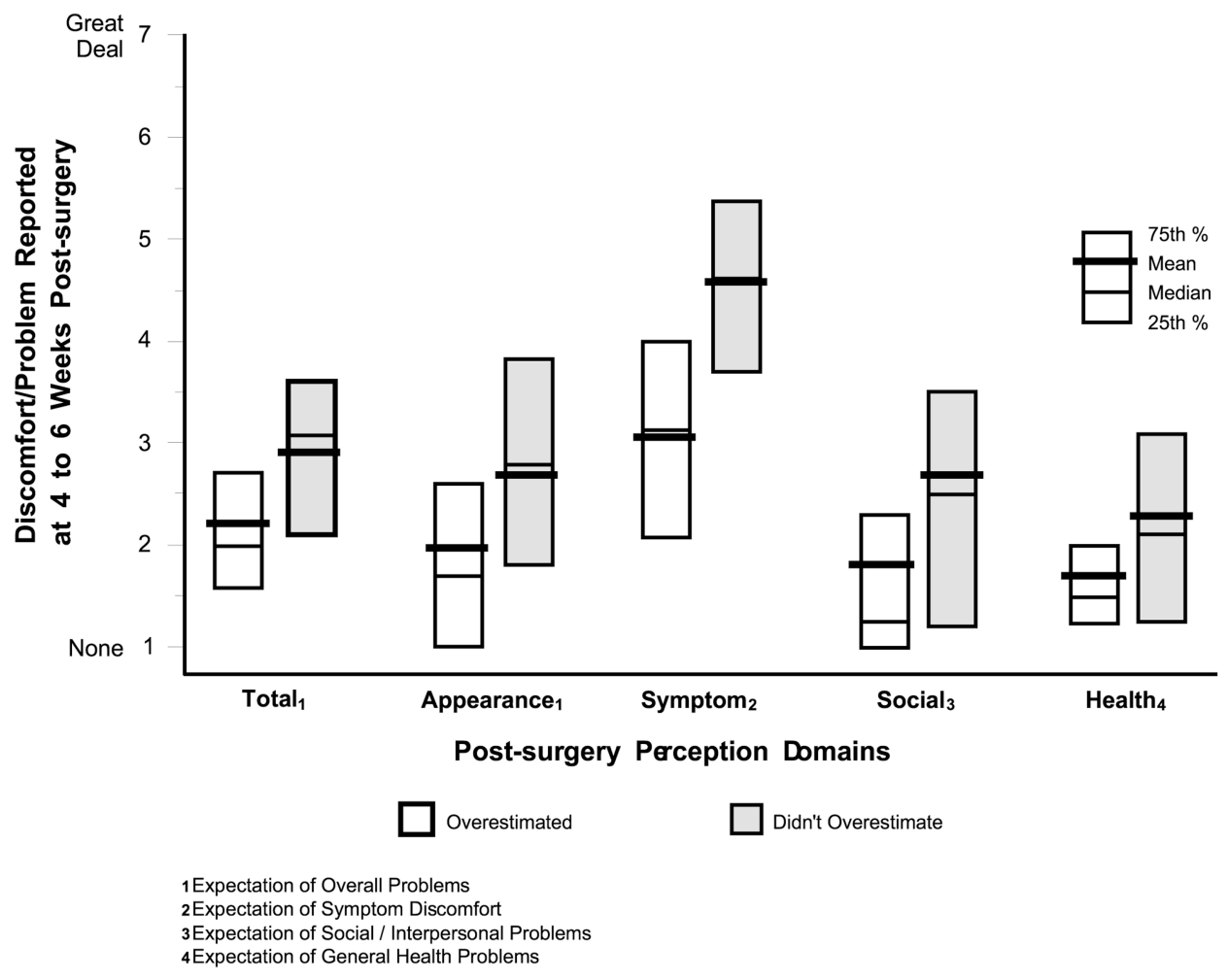

Figure 2.

Descriptive statistics for the overall level of perceived problems or discomfort experienced in the last month and each of the specific postsurgical domains, symptom burden, appearance, and social and general health concerns comparing those patients whose anticipatory level exceeded what they experienced and those who did not overestimate. Expectations of problems in social/interpersonal areas were compared with the experienced level of discomfort for both the social/interpersonal and the dentofacial appearance concerns at 4 to 6 weeks after surgery. 


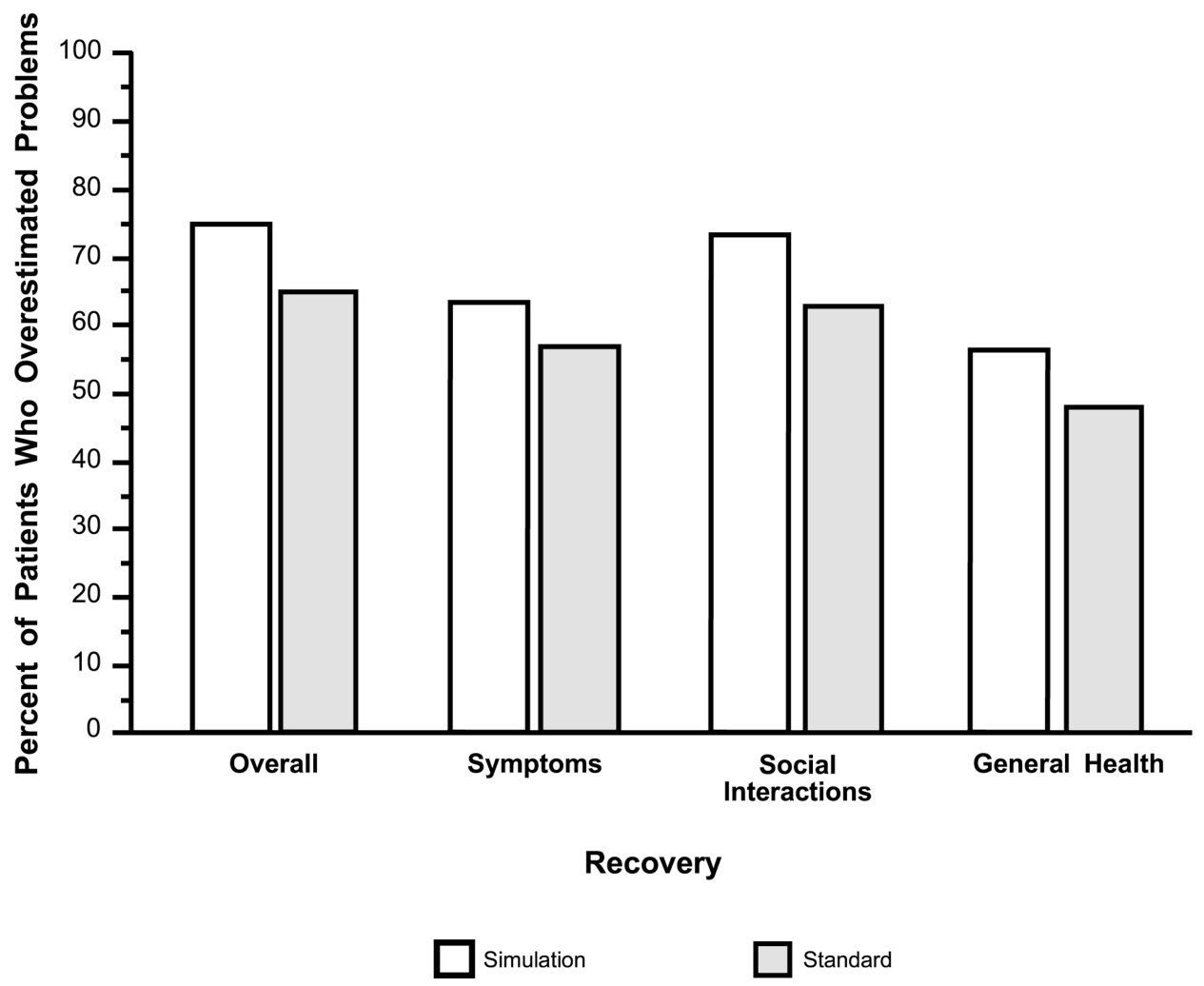

Figure 3.

Percentage of patients in the treatment simulation and standard preparation groups who overestimated the problems or concerns they would experience 4 to 6 weeks after surgery. 


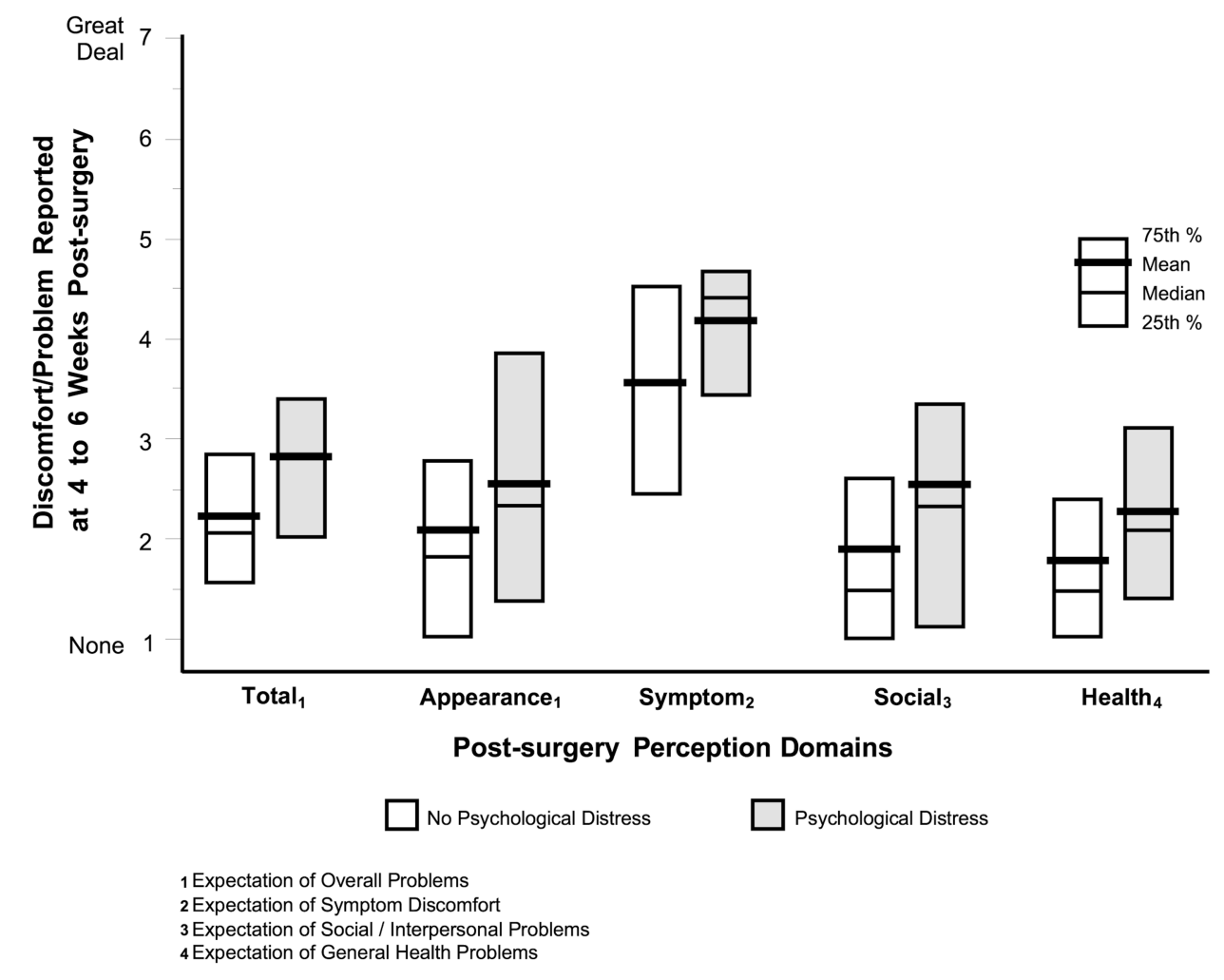

Figure 4.

Descriptive statistics for the overall level of perceived problems or discomfort experienced in the last month and for each of the specific postsurgical domains, symptom burden, appearance, and social and general health concerns, for those patients who were psychologically distressed (positive diagnosis) and those who were not. 
Table 1

Descriptions and Abbreviations Used for Patient Self-Report Questionnaires

\begin{tabular}{lll} 
Questionnaire & Construct & Data Collection Time \\
\hline $\begin{array}{l}\text { Symptom Checklist-90 Revised } \\
\text { (SCL-90R) }\end{array}$ & Current psychologic well-being & $\begin{array}{l}\text { Presurgery before randomization; } 4 \text { to 6 weeks after } \\
\text { surgery }\end{array}$ \\
$\begin{array}{ll}\text { Short-Term Expectations (STE) } \\
\text { Postsurgical Perceptions (PSP) }\end{array}$ & $\begin{array}{l}\text { Expectations of recovery burden } \\
\text { Presurgery after randomization (1 to 2 weeks) }\end{array}$ \\
Satisfaction (SAT) & Perceived recovery burden & 4 to 6 Weeks after surgery \\
\hline
\end{tabular}




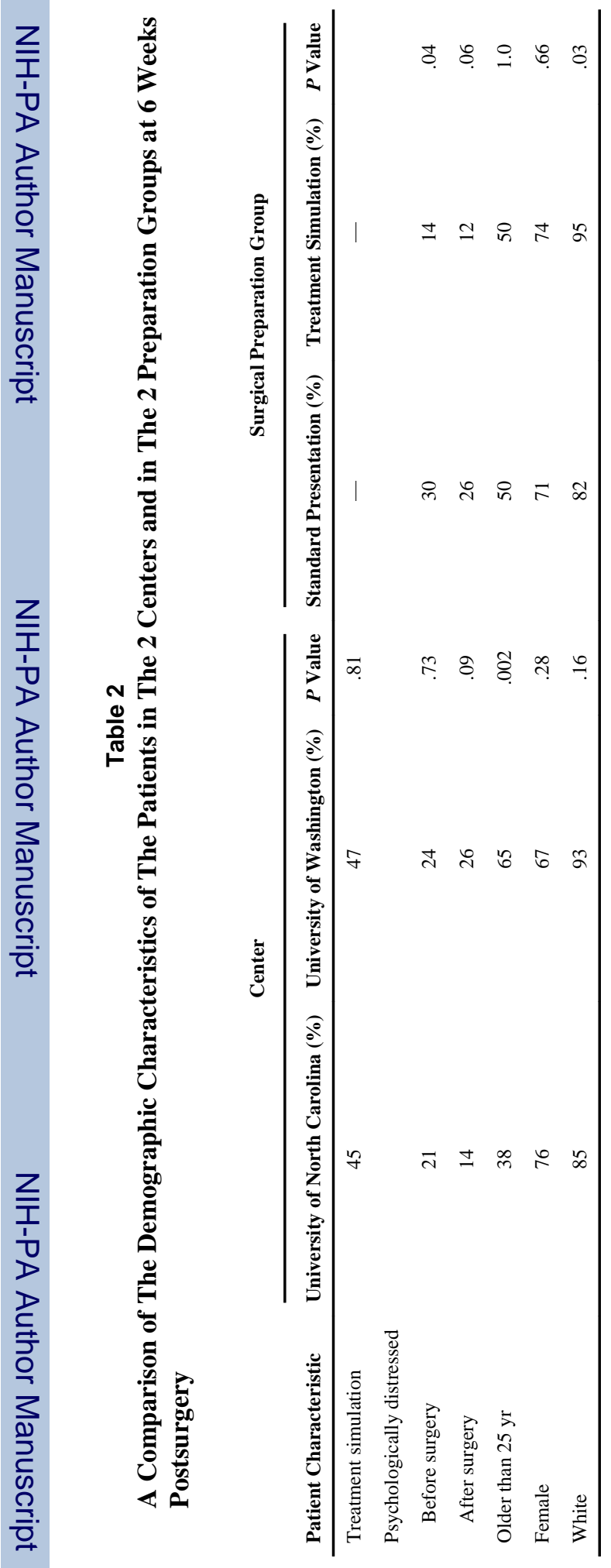




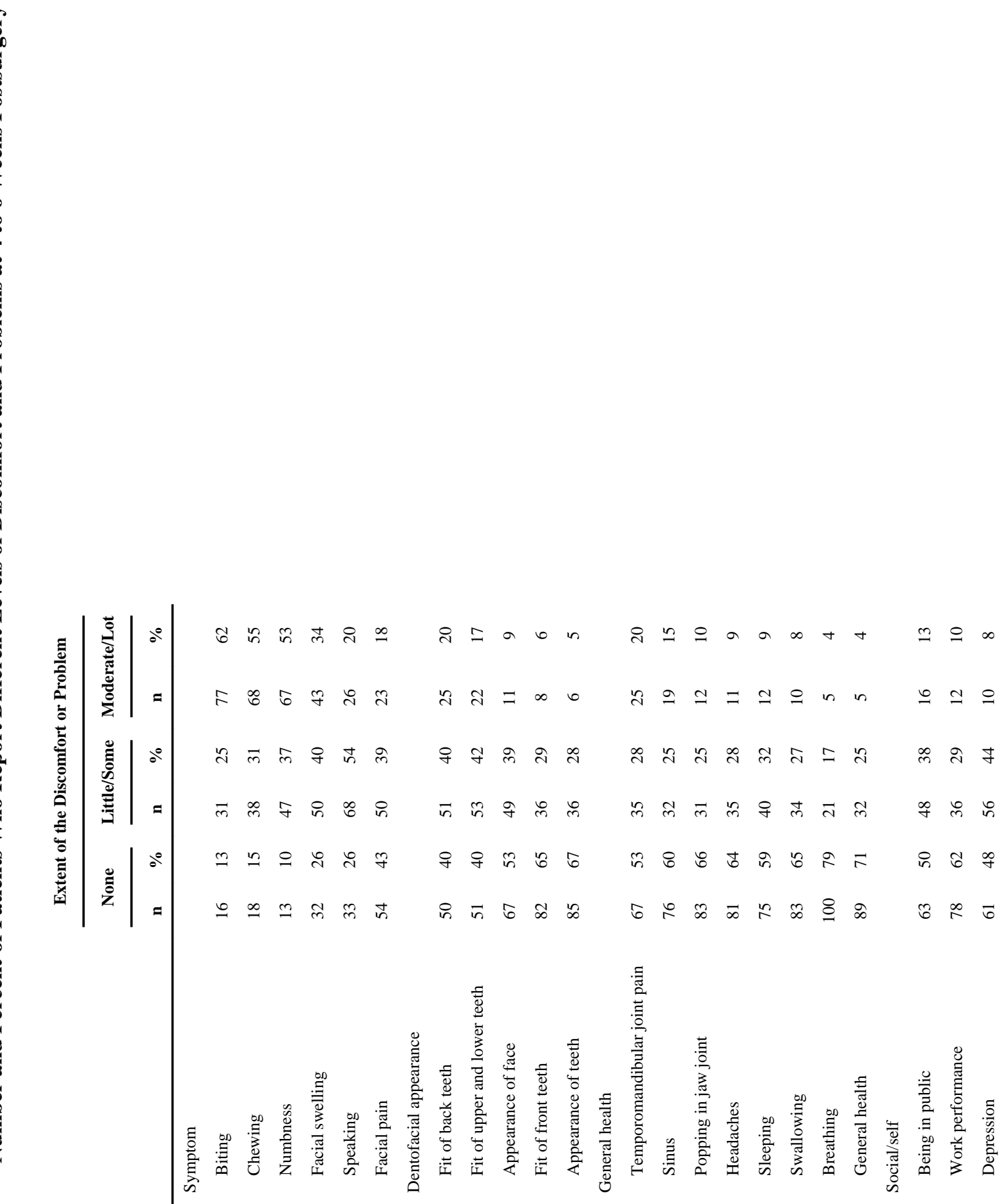




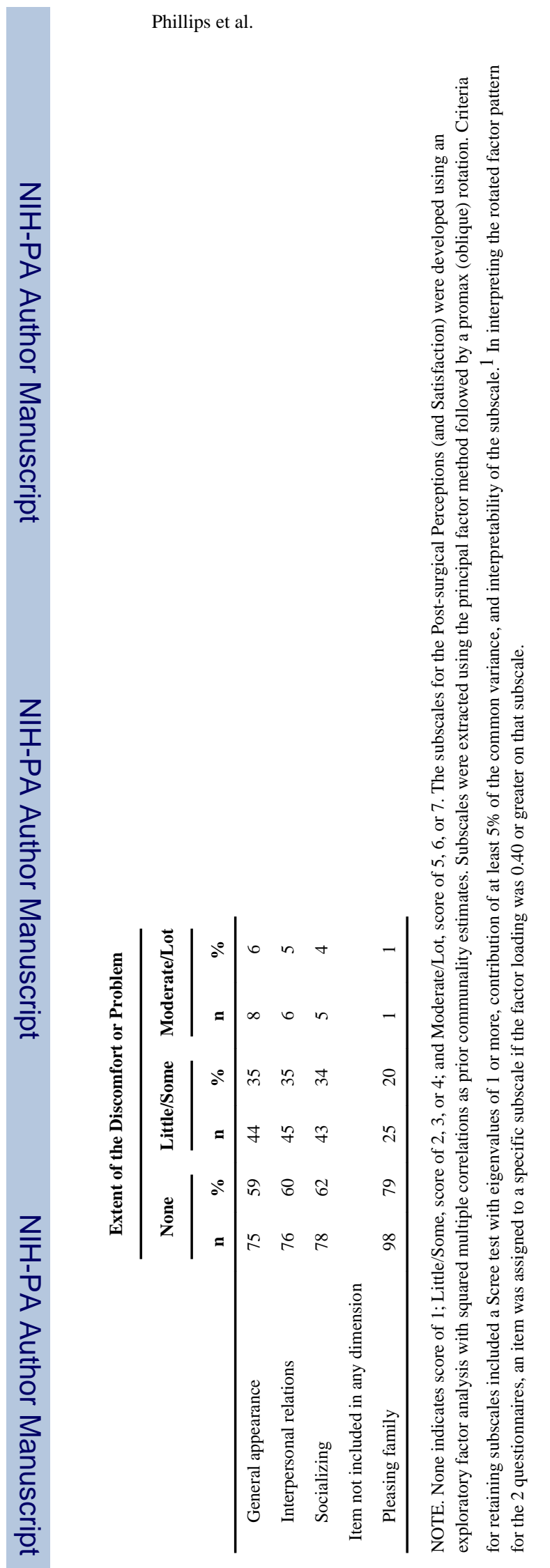

J Oral Maxillofac Surg. Author manuscript; available in PMC 2013 March 11. 


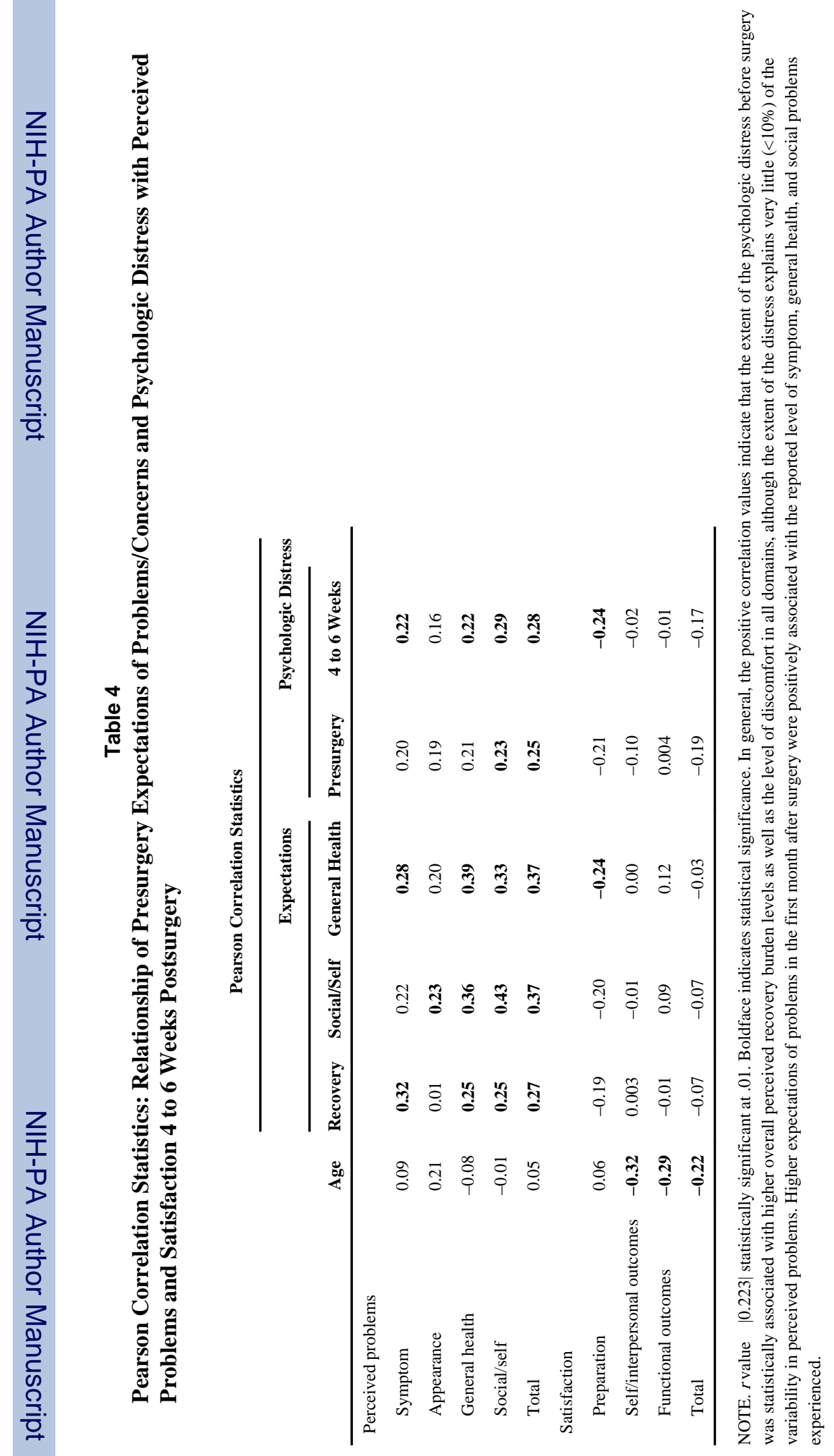

J Oral Maxillofac Surg. Author manuscript; available in PMC 2013 March 11. 


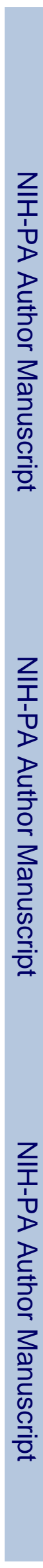

Phillips et al.

Page 20

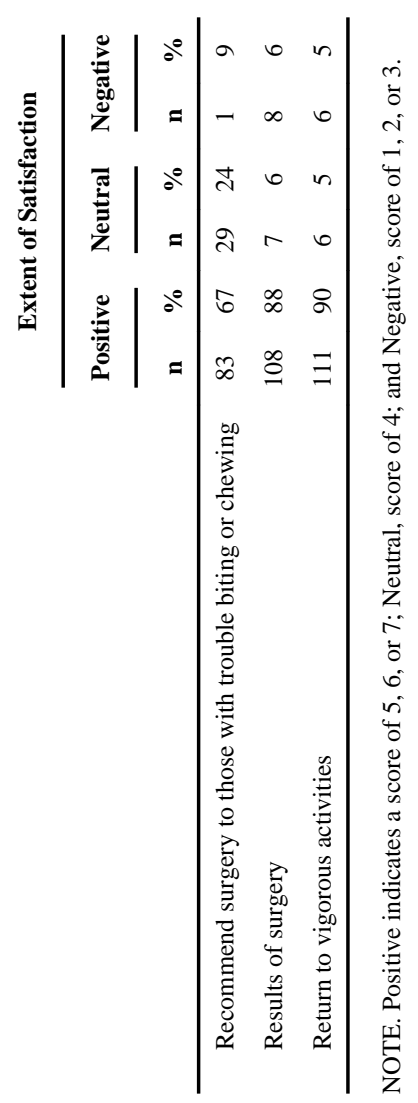

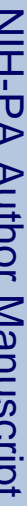

J Oral Maxillofac Surg. Author manuscript; available in PMC 2013 March 11. 
Table 6

Relationship of Perceived Problems and Satisfaction Indicated by Pearson Correlation Statistics 4 To 6 Weeks Postsurgery

\begin{tabular}{lcccc} 
& \multicolumn{5}{c}{ Satisfaction } \\
\cline { 2 - 6 } Perceived Problems & Self/Interpersonal Outcomes & $\begin{array}{c}\text { Preparation for Surgery and } \\
\text { Recovery }\end{array}$ & Functional Outcomes & Total Satisfaction \\
\hline Symptoms & $\mathbf{- 0 . 2 2}$ & $\mathbf{- 0 . 3 6}$ & $\mathbf{- 0 . 3 3}$ & $\mathbf{- 0 . 4 0}$ \\
Dentofacial Appearance & $\mathbf{- 0 . 3 1}$ & -0.16 & -0.18 & $-\mathbf{0 . 3 5}$ \\
General Health & -0.03 & $\mathbf{- 0 . 3 2}$ & -0.18 & $\mathbf{- 0 . 2 5}$ \\
Social/Self & $\mathbf{- 0 . 2 3}$ & $\mathbf{- 0 . 4 6}$ & -0.10 & $\mathbf{- 0 . 4 0}$ \\
\hline
\end{tabular}

NOTE. $r$ value $\geq|0.223|$ statistically significant at .01 . Boldface indicates statistical significance. In general, the negative correlation values indicate that the higher the perceived level of discomfort at 4 to 6 weeks, the lower is the satisfaction. Although statistically significant, the relationship between perceived level of discomfort and satisfaction explains at most $17 \%$ of the variability. 


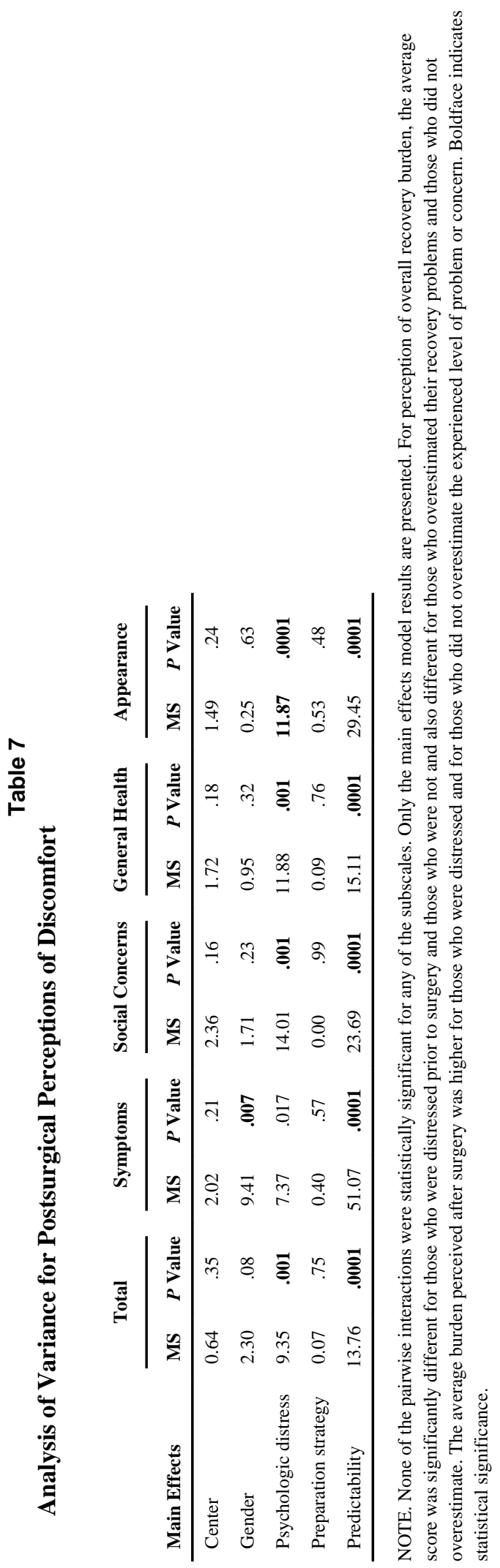




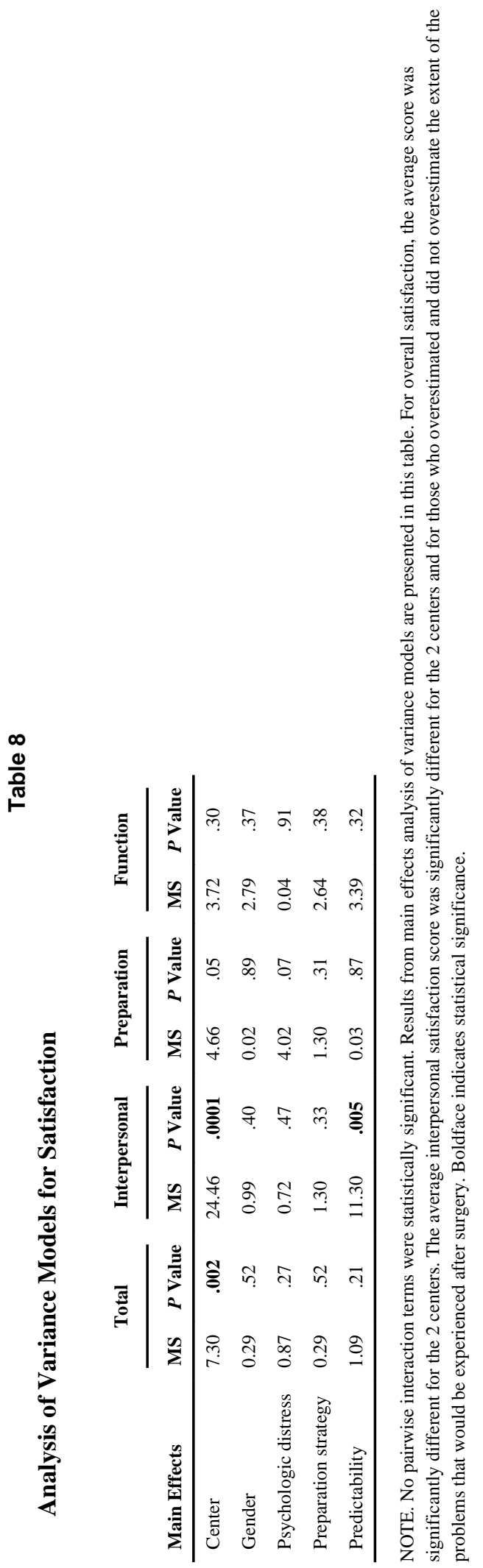

J Oral Maxillofac Surg. Author manuscript; available in PMC 2013 March 11. 